\title{
Cross-sections of residual nuclei from deuteron irradiation of thin thorium target at energy $7 \mathrm{GeV}$
}

\author{
Radek Vespalec ${ }^{1,2, a}$, Jindrich Adam ${ }^{1,3}$, Anton Alexandrovich Baldin ${ }^{1,5}$, Jurabek Khushvaktov ${ }^{1}$, \\ Alexander Alexandrovich Solnyshkin ${ }^{1}$, Vsevolod Mikhailovich Tsoupko-Sitnikov ${ }^{1}$, \\ Sergey Ivanovich Tyutyunikov ${ }^{1}$, Jitka Vrzalova ${ }^{1,3}$, Lukas Zavorka ${ }^{1,2}$, and Miroslav Zeman ${ }^{1,4}$ \\ 1 Joint Institute for Nuclear Research, Joliot-Curie 6, 141980 Dubna, Russian Federation \\ 2 Faculty of Nuclear Sciences and Physical Engineering of the CTU in Prague, Brehova 7, 11519 Prague, Czech Republic \\ ${ }^{3}$ Nuclear Physics Institute of the CAS, Rez 130, 25068 Rez, Czech Republic \\ 4 Brno University of Technology, Antoninska 548/1, 60190 Brno, Czech Republic \\ 5 Institute for Advanced Studies “OMEGA", Universitetskaya 19, 141980 Dubna, Russian Federation
}

\begin{abstract}
The residual nuclei yields are of great importance for the estimation of basic radiation-technology characteristics (like a total target activity, production of long-lived nuclides etc.) of accelerator driven systems planned for transmutation of spent nuclear fuel and for a design of radioisotopes production facilities. Experimental data are also essential for validation of nuclear codes describing various stages of a spallation reaction. Therefore, the main aim of this work is to add new experimental data in energy region of relativistic deuterons, as similar data are missing in nuclear databases. The sample made of thin natural thorium foil was irradiated at JINR Nuclotron accelerator with a deuteron beam of the total kinetic energy $7 \mathrm{GeV}$. Integral number of deuterons was determined with the use of aluminum activation detectors. Products of deuteron induced spallation reaction were qualified and quantified by means of gamma-ray spectroscopy method. Several important spectroscopic corrections were applied to obtain results of high accuracy. Experimental cumulative and independent cross-sections were determined for more than 80 isotopes including metastable isomers. The total uncertainty of results rarely exceeded $9 \%$. Experimental results were compared with MCNP6.1 Monte-Carlo code predictions. Generally, experimental and calculated cross-sections are in a reasonably good agreement, with the exception of a few light isotopes in a fragmentation region, where the calculations are highly under-estimated. Measured data will be useful for future development of high-energy nuclear codes. After completion, final data will be added into the EXFOR database.
\end{abstract}

\section{Introduction}

The Nuclotron accelerator of Veksler and Baldin Laboratory of High-Energy Physics at Joint Institute for Nuclear Research is able to provide relativistic deuteron beams up to $8 \mathrm{GeV}$ per nucleon and intensity up to $10^{10}$ particles per cycle [1]. Several experiments were performed using various spallation targets in a frame of Energy and Transmutation of Radioactive Waste (E\&T RAW) collaboration formed in the Joint Institute for Nuclear Research in Dubna (http://et.jinr.ru) in order to investigate possibilities of so called Relativistic Nuclear technologies (RNT) in the accelerator driven systems concept (ADS).

Stochastic transport codes like MCNP [2] serve as an irreplaceable tools for planning experiments at complex spallation set-ups. In the case of lower energies, the MCNP6 code uses so called data libraries (tables of evaluated nuclear data). However, in the case of accelerator driven systems, the range of needed nuclear data is extended up to several $\mathrm{GeV}$. Therefore, for higher particle energies above $150 \mathrm{MeV}$, the code uses nuclear models, sometimes referred as high-energy event generators.

a e-mail: vespalec@jinr.ru
Results from integral benchmark experiments performed at massive natural uranium target QUINTA shows, that different event generators play an important role in final agreement between experimental and calculated quantities $[3,4]$.

Experimental nuclear data are essential for validation and future improvement of nuclear codes describing various stages of spallation reaction. However, necessary data are often missing in the EXFOR database [5] like for the ${ }^{232} \mathrm{Th}(\mathrm{d}, *)$ in the case of energies higher than $0.5 \mathrm{GeV}$.

The main object of the current project is to obtain new experimental data from irradiation of thin thorium and uranium targets using the direct kinematics method. The secondary objective is to perform a test of the aforementioned codes used for a description of spallation reaction in large-scale spallation set-ups.

\section{Experimental methods}

\subsection{Irradiation}

A stack of thorium foils was irradiated with $7 \mathrm{GeV}$ deuteron beam at the experimental area F3 at extracted beams of the Nuclotron accelerator complex. The sample consisted of three pure thorium foils $\left(141 \mathrm{mg} / \mathrm{cm}^{2}\right)$ 


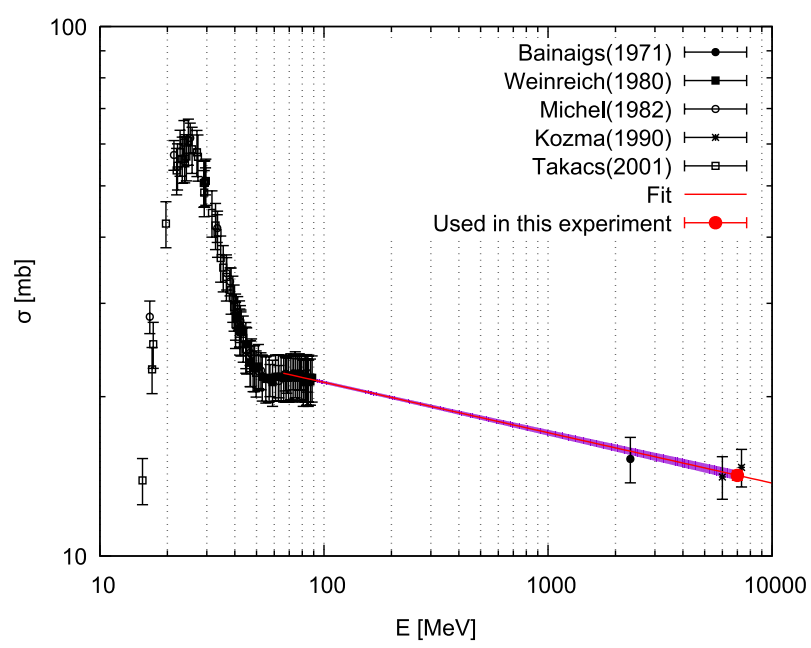

Figure 1. Experimental cross-sections for the ${ }^{27} \mathrm{Al}(\mathrm{d}, 3 \mathrm{p} 2 \mathrm{n}){ }^{24} \mathrm{Na}$ reaction obtained from the EXFOR database together with extrapolation fit and the cross-section value used in this experiment.

surrounded by aluminum foils $\left(106 \mathrm{mg} / \mathrm{cm}^{2}\right)$. Foils were wrapped in a paper in order to minimize a migration of residual nuclei out of the stack.

The ${ }^{27} \mathrm{Al}(\mathrm{d}, \mathrm{x}){ }^{24} \mathrm{Na}$ beam monitor reaction was used to calculate an integrated deuteron beam flux. Reaction's cross-section was obtained as an interpolation of values published in EXFOR database [5]. Regrettably, only few sets of data are available in $\mathrm{GeV}$ energy region, namely one point at $2.33 \mathrm{GeV}$ determined by Bainaigs (15.25 \pm $1.50) \mathrm{mb}[6]$ and two points by Kozma [7]: (14.1 \pm 1.3$) \mathrm{mb}$ at $6.0 \mathrm{GeV}$ and $(14.7 \pm 1.2) \mathrm{mb}$ at $7.3 \mathrm{GeV}$ by using absolute calibrated fission chambers. In the low energy region up to $60 \mathrm{MeV} 3$ data sets were chosen, namely Weinreich [8], Michel [9] and Takacs [10].

Experimental data were fitted by a function $f(E)=$ $10^{a+b \log (E)}$. The experimental values retrieved from EXFOR database together with function $f(E)$ are shown in Fig. 1. The cross-section value for $7 \mathrm{GeV}$ was estimated to $13.8 \mathrm{mb}$. Detailed description of the fitting process and data selection metodology can be found in [11] and [12]. The cross-section uncertainty was not incorporated into the total integral beam uncertainty due to the lack of information concerning the uncertainty propagation. According to the aluminum monitors, the thorium sample accepted a total beam integral $3.90 \cdot 10^{11}$ deuterons.

\subsection{Gamma spectroscopy}

After irradiation, the samples were measured by using a high resolution $\gamma$ spectrometer in a YaSNaPP spectroscopy complex of the Dzhelepov Laboratory of Nuclear Problems (DLNP). The $\gamma$ ray spectrometer was a p-type coaxial Canberra high-purity germanium (HPGe) detector with a diameter of $58 \mathrm{~mm}$ and a thickness $55 \mathrm{~mm}$. The relative efficiency of the detector at $1.33 \mathrm{MeV}$ was about $34.7 \%$ and the energy resolution $1.76 \mathrm{keV}$ at full width half maximum (FWHM).

Due to a necessary transportation between the irradiation facility and the spectroscopy complex, first measurements started one hour after the end of irradiation. The sample was measured in a stable geometry and measuring times varied from 20 minutes up to several days.
Full-energy-peak and total efficiency calibration was performed before the start of the experiment using a set of certified calibration standards with relative uncertainty $3 \%$. The detector was surrounded by a standard shielding consisting of a $10 \mathrm{~cm}$ thick lead bricks together with additional cadmium and copper plates.

The HPGe-detector was coupled to a DSA-1000 digital multichannel analyzer connected to a computer with Canberra Genie-2000 spectroscopic software. This system served for gamma spectra acquisition.

Analysis of measured gamma spectra was done by using the gamma-spectrometry software DEIMOS32 [13]. The program is able to process gamma spectra in fully manual mode. This is quite usefull especially in the case of rich spectra with significant number gamma lines and multiplets. The total number of fitted gamma-lines in each spectrum was gradually increasing from 160 peaks in the first spectrum up to 300 peaks in the last one.

Data analysis was performed with the use of in-house developed YaSNaPP-2 code (unpublished). The following set of spectroscopic corrections was used to correct the raw data: self absorption corr., coincidence-summing correction (TrueCoinc software [14]), real coincidence corr., detector efficiency and dead time corr., and the beam instability correction.

\subsection{Cross-section calculation}

Generally, a hundreds of isotopes are produced during irradiation. However, only limited amount of them can be identified using the off-line gamma spectroscopy. In the case of presented experiment, we were able to measure isotopes with half-lives longer than 30 minutes due to a distance between the irradiation facility and the spectroscopy laboratory. The decay of chained residual nuclei $a \stackrel{\lambda_{a}}{\rightarrow} b \stackrel{\lambda_{b}}{\rightarrow} c$ formed during irradiation is described with the following set of equations:

$$
\begin{gathered}
d N_{a}(t) / d t=Q_{a}-\lambda_{a} N_{a}(t) \\
d N_{b}(t) / d t=Q_{b}-\lambda_{b} N_{b}(t)+\lambda_{a} N_{a}(t)
\end{gathered}
$$

where $Q_{a}, Q_{b}$ are formation rates for nuclei a and b.

Solving these equations, corresponding independent cross-sections can be calculated according to the following formulas:

$$
\begin{gathered}
\sigma_{a}=\frac{\lambda_{a}\left(\lambda_{b}-\lambda_{a}\right)}{K_{b} \lambda_{b}\left(1-e^{-\lambda_{a} \cdot t_{i r r}}\right)} \cdot A \\
\sigma_{b}=\frac{\lambda_{b}}{K_{b}\left(1-e^{-\lambda_{b} \cdot t_{i r r}}\right)} \cdot B+\frac{\lambda_{a}^{2}}{K_{b} \lambda_{b}\left(1-e^{-\lambda_{b} \cdot t_{i r r}}\right)} \cdot A \\
K_{b}=N_{p} \cdot N_{m} \cdot I_{\gamma}
\end{gathered}
$$

where $N_{P}$ is the flux of particles incident on the target, $N_{m}$ is the number of nuclei per $\mathrm{cm}^{2}$ at the surface of the target, $t_{i r r}$ is a time of irradiation, $\lambda_{i}$ is a decay constant and $I_{\gamma}$ is an intensity of gamma line with energy $E_{\gamma}$. These data were extracted from the ENSDF database [15]. Parameters $A$ and $B$ were estimated by least-squares fitting the decay curve from several measurements:

$$
\begin{aligned}
S_{\gamma}\left(E_{\gamma, i}\right)= & \left\{A e^{-\lambda_{a} t_{\text {dec }}}\left(1-e^{-\lambda_{a} t_{\text {real }}}\right)\right. \\
& \left.+B e^{-\lambda_{b} t_{\text {dec }}}\left(1-e^{-\lambda_{b} t_{\text {real }}}\right)\right\},
\end{aligned}
$$




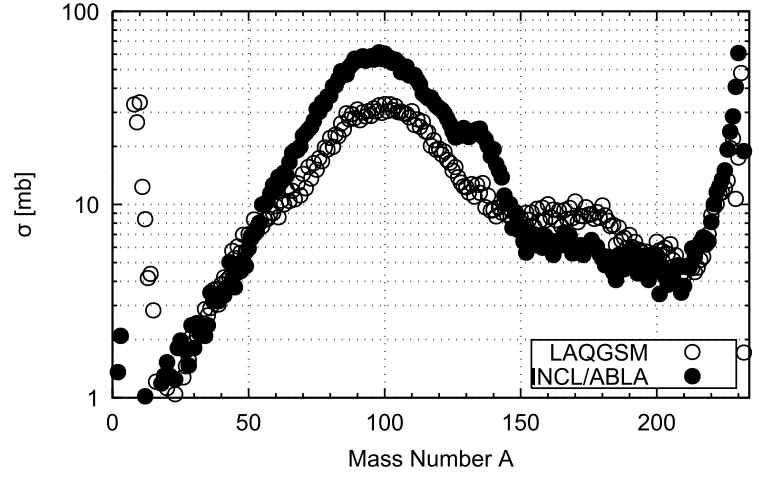

Figure 2. Comparison of the mass distributions of produced residues in the spallation process in natural thorium initiated by $7 \mathrm{GeV}$ deuterons simulated with LAQGSM03.03 and INCL4.2/ABLA event generators employed in the MCNP6 code.

where $t_{d e c}$ is a time interval between the end of irradiation and the start of the measurement, $t_{\text {real }}$ and $t_{\text {live }}$ are real and live time of the measurement. Detailed description of derivation of equations can be found in a paper [16].

Total uncertainty was combined from statistical and systematic errors according to the Gaussian law of error propagation. The systematic uncertainty is composed of the detector calibration uncertainty with the relative uncertainty in activity of $3 \%$, the beam integral uncertainty $\approx 6 \%$ (uncertainty of monitor's crosssection was excluded, see Sect. 2.1) and the sample mass uncertainty $\approx 0.1 \%$. Nuclear data uncertainties were incorporated during the data analysis. The statistical uncertainty rarely exceeded $5 \%$ due to a sufficient counting statistics.

\subsection{Computational predictions}

MCNP6 is a general multipurpose transport code based on a Monte-Carlo method used to simulate various nuclear applications [2]. Besides neutron flux and criticality calculations, the code is also able to compute residual nuclei yields from high-energy collisions where appropriate evaluated nuclear data libraries are missing. Measured cross-sections were compared with predictions of the MCNP6 using two different event generators available to simulate high-energy nuclear reactions:

- Los Alamos version of the Quark Gluon-String Model (LAQGSM03.03) used for incident particles with energies above $4.5 \mathrm{GeV}$ [17].

- The Intra-Nuclear Cascade model (INCL4.2) [18, 19] merged with the evaporation-fission model ABLA (version KHSV3p) [20].

All used event generators compute only direct formation of individual radio-nuclides. The direct formation is described with the term "independent" (i), the formation by the decay of parents is called "cumulative" (c). Cumulative cross-sections were calculated using independent values summed separately according to the decay schemes using the Table of Isotopes $\left(8^{\text {th }}\right.$ edition) [21] and branching ratios $v_{k}$ retrieved from ENSDF database [15].
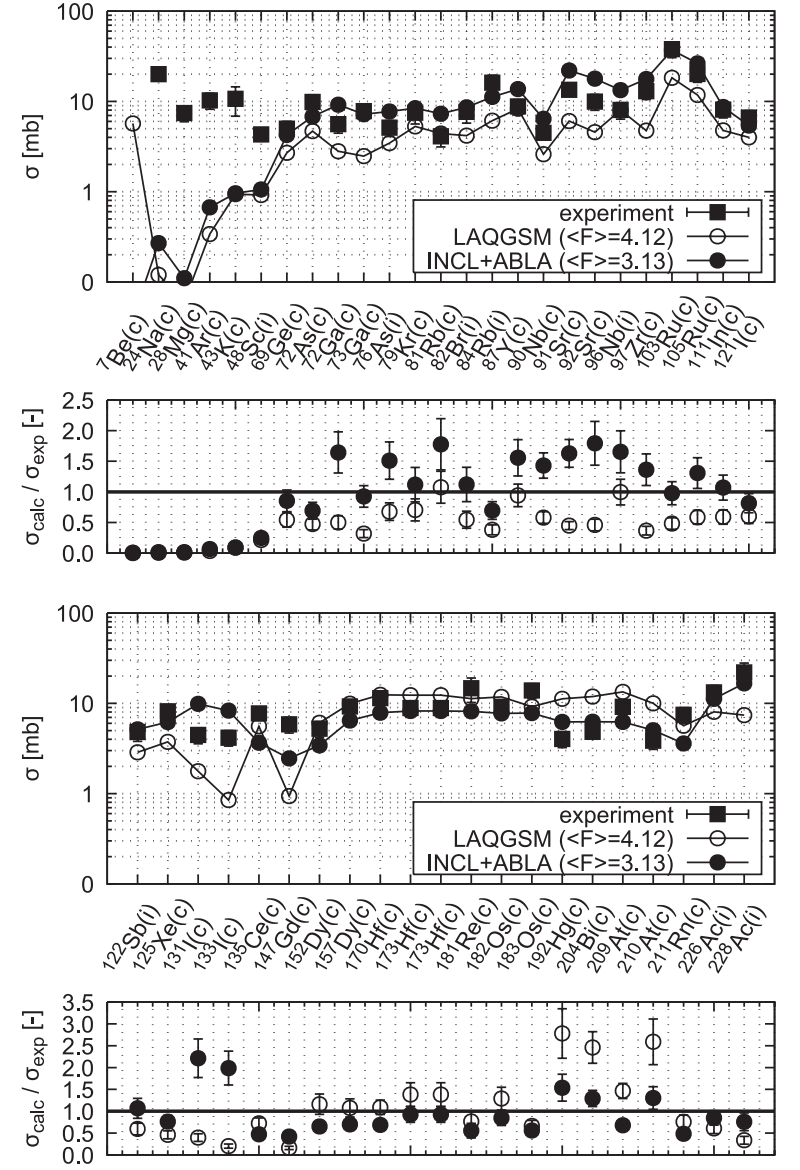

Figure 3. Detailed comparison between selected measured cross-sections and the predictions of LAQGSM03.03 and INCL4.2/ABLA event generator of MCNP6 in order of increasing mass. (i) Stands for independent and (c) for cumulative cross-section.

\subsection{Comparison between experimental results and theoretical predictions}

Figure 3 shows a detailed comparison between experimental and predicted results. The MCNP6 transport code is not capable to calculate metastable states, therefore measured products in metastable states $\left({ }^{82 \mathrm{~m}} \mathrm{Br},{ }^{84 \mathrm{~m}} \mathrm{Rb},{ }^{85 \mathrm{~m}} \mathrm{Kr},{ }^{101 \mathrm{~m}} \mathrm{Rh}\right.$, $\left.{ }^{111 \mathrm{~m}} \mathrm{Pd},{ }^{130 \mathrm{~m}} \mathrm{I}\right)$ were discharged from the comparison.

Generally, selected residual nuclei are in good agreement for both spallation $(\mathrm{A}>200)$ and fission regions $(50<\mathrm{A}<150)$ with the exception of the region with the lowest mass numbers $(\mathrm{A}<50)$, where only a few isotopes could be identified $\left({ }^{24} \mathrm{Na},{ }^{28} \mathrm{Mg},{ }^{41} \mathrm{Ar},{ }^{43} \mathrm{~K}\right)$ with the use of gamma ray spectroscopy. In this region, a disagreement reaches several magnitudes.

Therefore, more detailed investigation is needed. One of the possibilities, how to improve agreement between calculated and predicted cross-sections is of usage of new versions of the event generators, like LAQGSM03.03F [22] or INCL4.6 [23] which have not been incorporated into MCNP6 code yet.

\section{Results and conclusion}

Thin thorium foils were irradiated at Nuclotron accelerator with a deuteron beam of $7 \mathrm{GeV}$. Till now, 86 isotopes of spallation and fission products including metastable 
states were identified. Partial cross sections were calculated using the activation technique. Experimental data were compared with theoretical calculations by LAQGSM.03.03 and INCL4.2 event generators of the MCNP6 transport code. The comparison shows that experimental and theoretical values are in a reasonably good agreement. In the most cases, the experimental values lay between LAQGSM.03.03 and INCL4.2/ABLA event generators. The greatest disagreement between codes and measured values exists in the region with mass number $\mathrm{A}<50$. In this region, disagreement between calculated and measured data approaches an order of magnitude. Further experiments would improve understanding of the mechanism of these reactions and will make further improvements of the theoretical models possible.

The authors would like to thank the staff of the Nuclotron accelerator in JINR for great support. This work was supported by the grant of " $3+3$ " cooperation between the Czech Republic and JINR.

\section{References}

[1] A. Kovalenko et al., Nuclotron at JINR: Operation Experience and Recent Development, in Proceedings, 13th Heavy Ion Accelerator Technology Conference (HIAT2015): Yokohama, Japan, September 7-11, 2015, MOPA19 (2016)

[2] T. Goorley, M. James, T. Booth, F. Brown, J. Bull, L. Cox, J. Durkee, J. Elson, M. Fensin, R. Forster et al., Nuclear Technology 180, 298 (2012)

[3] L. Zavorka, J. Adam, M. Artiushenko, A. Baldin, V. Brudanin, O. Bukhal, P. Caloun, V. Chilap, W. Furman, K. Husak et al., Annals of Nuclear Energy 80, 178 (2015)

[4] J. Khushvaktov, J. Adam, A. Baldin, V. Chilap, V. Furman, F. Sagimbaeva, A. Solnyshkin, V. Stegailov, P. Tichy, V. Tsoupko-Sitnikov et al., Nuclear Instruments and Methods in Physics Research Section B: Beam Interactions with Materials and Atoms 381, 84 (2016)

[5] N. Otuka, E. Dupont, V. Semkova, B. Pritychenko, A. Blokhin, M. Aikawa, S. Babykina, M. Bossant, G. Chen, S. Dunaeva et al., Nuclear Data Sheets 120, 272 (2014)

[6] J. Banaigs, J. Berger, J. Duflo, L. Goldzahl, O. Harff, M. Cottereau, F. Lefebvres, H. Quechon,
P. Tardy-Joubert, Nuclear Instrum.and Methods in Physics Res. 95, 307 (1971)

[7] P. Kozma, V.V. Yanovsky, Czechoslovak Journal of Physics 40, 393 (1990)

[8] R. Weinreich, H. Probst, S. Qaim, The International Journal of Applied Radiation and Isotopes 31, 223 (1980)

[9] R. Michel, G. Brinkman, R. Galas, R. Stuck (1982), EXFOR No. A0158

[10] S. Takacs, F. Szelecsenyi, F. Tarkanyi, M. Sonck, A. Hermanne, Y. Shubin, A. Dityuk, M. Mustafa, Z. Youxiang, Nuclear Instruments and Methods in Physics Research Section B: Beam Interactions with Materials and Atoms 174, 235 (2001)

[11] J. Adam, A. Baldin, V. Chilap, W. Furman, K. Katovsky, J. Khushvaktov, V. Kumar, V. Pronskikh, I. Marin, A. Solnyshkin et al., Physics Procedia 80, 94 (2015)

[12] M. Suchopar, J. Adam, P. Chudoba, O. Svoboda, J. Vrzalova, V. Wagner (2012), internal report

[13] J. Frana, Journal of Radioanalytical and Nuclear Chemistry 257, 583 (2003)

[14] S. Sudar, IAEA-TECDOC-1275, 37-48 (2002)

[15] Evaluated nuclear structure data file (ENDSF) retrieval, national Nuclear Data Center, Brookhaven National Laboratory, http://www.nndc.bnl.gov/ensdf /

[16] I. Adam, J. Mrazek, J. Frana, A.R. Balabekyan, V. Pronskikh, V. Kalinnikov, A. Priemyshev, Measurement Techniques 44, 93 (2001)

[17] S.G. Mashnik, K.K. Gudima, R.E. Prael, A.J. Sierk, M.I. Baznat, N.V. Mokhov (2008), arXiv:0805.0751

[18] A. Boudard, J. Cugnon, S. Leray, C. Volant, Phys. Rev. C 66, 044615 (2002)

[19] J. Cugnon, C. Volant, S. Vuillier, Nuclear Physics A 625, 729 (1997)

[20] A. Junghans, M. de Jong, H.G. Clerc, A. Ignatyuk, G. Kudyaev, K.H. Schmidt, Nuclear Physics A 629, 635 (1998)

[21] R.B. Firestone, Table of Isotopes, 2 Volume Set (Wiley-VCH, 1997), ISBN: 0471149187

[22] S.G. Mashnik, L.M. Kerby, K.K. Gudima, A.J. Sierk, J.S. Bull, M.R. James, Production of energetic light fragments in CEM, LAQGSM, and MCNP6 (2016), arXiv: 1607.02506

[23] A. Boudard, J. Cugnon, J.C. David, S. Leray, D. Mancusi, Phys. Rev. C 87, 014606 (2013) 